\title{
Distribution of coronal heating in a solar active region
}

\author{
C. Gontikakis, I. Contopoulos, and H. C. Dara
}

\author{
Research Center for Astronomy and Applied Mathematics, Academy of Athens, 4 Soranou Efessiou Str., Athens 11527, Greece \\ e-mail: cgontik@academyofathens.gr
}

Received 16 November 2007/ Accepted 17 June 2008

\begin{abstract}
Aims. We investigate the distribution of heating of coronal loops in a non-flaring solar active region, using a simple electrodynamic model: the random displacements of the loop footpoints, caused by photospheric plasma motions, generate electric potential differences between the footpoints and, as a result, electric currents flow along the loops, producing Ohmic heating.

Methods. We implement our model on the closed magnetic field lines in the potential magnetic field extrapolation of an MDI active region magnetogram. For each one of the magnetic field lines, we compute the heating function and obtain the hydrostatic distribution of temperature and pressure. We find that coronal heating is stronger close to the footpoints of the loops and asymmetric along them. We obtain scaling laws that relate both the mean volumetric heating to the loop length, and the heating flux through the loop footpoints to the magnetic field strength at the footpoints. Our results agree with observational data.

Results. According to our model, we attribute the observed small coronal-loop width expansion to both the preferential heating of coronal loops of small cross-section variation, and the cross-section confinement due to the random electric currents flowing along the loops.

Conclusions. We conclude that our model can be used as a simple working tool in the study of solar active regions.
\end{abstract}

Key words. Sun: corona - Sun: activity - Sun: magnetic fields

\section{Introduction}

Coronal loops are the main structures observed in the solar corona. To understand coronal heating, we must therefore understand how coronal loops are heated. One particular school of thought argues that the heating could originate from the braiding of magnetic field lines due to photospheric plasma motions (Parker 1972). According to this scenario, disturbed magneticfield lines release the excess of stored magnetic energy by means of small-scale reconnection events that heat the plasma, and appear as bright loops.

A significant part of the energy release could be due to Ohmic heating of DC electric currents. Interestingly, the observed heating rates (Withbroe \& Noyes 1977) cannot be reached if we consider Ohmic heating with an electrical resistivity based on Coulomb interactions of a fully ionized plasma (Spitzer 1962). Coronal plasma is a highly conducting medium, and such high heating rates can only be achieved on small spatial scales, such as inside reconnecting current sheets.

Coronal loop heating due to magnetic-field braiding can be simulated using resistive Magneto-Hydro-Dynamic (MHD) codes for a turbulent single loop (Rappazzo et al. 2007; Hendrix \& Van Hoven 2001; Dmitruk \& Gómez 1997, 1999) or an entire active region (Gudiksen \& Nordlund 2002, 2005a,b). Petrie (2006a) modelled an ensemble of loops with current sheets, using analytical solutions of ideal MHD. All such numerical approaches present an inherent difficulty: one should include, in the same simulation, length scales of the order of meters on which energy diffusion occurs, and length scales of thousands of kilometers that represent the size of coronal loops (Klimchuk 2006). We could argue, however, that in situations where a steady-state turbulent energy cascade from larger to smaller scales develops, the dissipation rate is independent of the smallest scales considered in the simulation.

A phenomenological approach to the coronal heating problem is to obtain scaling laws that relate different physical characteristics of loops, such as maximum temperature, pressure and length, with the heating along their length, under the assumption that the loops are steady. Such scaling laws were tested in loops observed by Skylab (Rosner et al. 1978a; Serio et al., 1981) and Yohkoh (Klimchuk \& Porter 1995) and in statistical samples of entire active regions (Golub et al. 1980; Fisher et al. 1998; Schrijver et al, 2004; Warren \& Winebarger 2006).

In the present work, we develop a simple electrodynamic model that reproduces qualitatively the main morphological features of coronal loop heating without the need to resort to complicated three-dimensional numerical MHD simulations. Our working assumption is that the loops are heated by direct current (DC) Ohmic dissipation, caused by electric currents that flow randomly along the loops. These currents are generated by magnetic field-line braiding, due to photospheric convective motions at the loop footpoints. The complex microphysics involved in the heating mechanism, such as reconnection, current cascades and/or turbulence (see Aschwanden 2005, for a review), are modelled by means of an enhanced effective electric resistivity. Our aim is to investigate the distribution of coronal heating in an observed active region and compute scaling laws that can be compared with observations.

We begin with a description of our model in Sect. 2. In Sect. 3, we apply it to a particular observed active region and obtain various scaling laws. In Sect. 4, we study the hydrostatic atmosphere above the active region. Our conclusions are obtained in Sect. 5. 


\section{Model description}

We base our discussion on the simplest possible average coronal magnetic-field configuration, that of a current-free magnetic field, where

$\nabla \times \boldsymbol{B} \approx 0$.

Such a magnetic-field configuration is characterized as potential. It has been shown in the literature (e.g. Gudiksen \& Nordlund 2005a) that this is an acceptable first order approximation for several relaxed (i.e. in quasi-equilibrium) active regions. As we see, although electric currents develop along coronal loops, they have random directions, so that Eq. (1) remains, on average, a valid approximation. To zeroth order, this allows us to simulate the 3D magnetic structure of any particular active region by extrapolating the photospheric magnetic field obtained in its magnetogram.

At each photospheric point corresponds a value of the vertical magnetic field $B_{0 \perp}$ and a random horizontal photospheric velocity due to granulation $v \sim \pm 1 \mathrm{~km} \mathrm{~s}^{-1}$. This configuration leads to the development of random horizontal electric fields $E \sim B_{0 \perp} v / c$, varying over the characteristic velocity turnover scale $l$ of the order of $\sim 1000 \mathrm{~km}$. This is equivalent to the development of a random photospheric electric potential distribution

$V \sim \frac{B_{0 \perp} v l}{c}$

at the coronal loop footpoints, where $c$ is the speed of light. The main idea in our model is that the coronal magnetic-field loops provide a conductive path that discharges the random electric potential differences between the two loop footpoints. Obviously, this yields random electric currents along the loops that we now calculate.

In a quasi-stationary configuration, the kinetic energy of random photospheric motions is continuously channelled through these random electric currents to the heating of the coronal loop. We may therefore model the complex microphysics of the heating mechanism by means of an effective electric resistivity $\eta_{\mathrm{e}}$ of the coronal plasma; each coronal loop of length $L$ and cross section along the loop $S$ then develops an effective integrated resistance

$R=\int_{s=0}^{L} \eta_{\mathrm{e}} \frac{\mathrm{d} s}{S}$

where, $s$ is the distance along the loop. The integration is taken along the loop length from one footpoint to the other. Due to the constancy of the magnetic flux along each individual loop, the cross section can be derived as a function of the magnetic field $B$ along the loop as

$S=B_{0} S_{0} / B$

where $B_{0}$ and $S_{0}$ are the magnetic-field strength and cross section, respectively, at one footpoint, i.e. at $s=0$.

The above parametrization allows us to estimate the random electric currents flowing along each coronal loop above an active region as

$I=\frac{\delta V}{R}$,

where $\delta V \sim V$ is the random electric potential difference between the two loop footpoints as determined by Eq. (2). We estimate the volumetric Ohmic heating at each position along the loop as

$h=\eta_{\mathrm{e}} j^{2} \equiv \eta_{\mathrm{e}}\left(\frac{I}{S}\right)^{2} \sim \eta_{\mathrm{e}} \frac{B_{0 \perp}^{2} v^{2} l^{2}}{c^{2} R^{2} S^{2}}$ where $j$ is the electric current density. The magnitude of the effective electric resistivity is obtained from coronal observations in the framework of the Ohmic dissipation heating mechanism. Observations of coronal energy losses (Withbroe \& Noyes 1977; Aschwanden 2005, p. 366) imply a volumetric heating rate for a loop with length $L$ of the order of

$$
E=2 \times 10^{-3} L_{10}^{-1}\left(\mathrm{erg} \mathrm{cm}^{-3} \mathrm{~s}^{-1}\right)
$$

This implies that the corresponding current density for Ohmic dissipation is of the order of

$j=\left(E / \eta_{\mathrm{e}}\right)^{1 / 2}=1.4 \times 10^{7} L_{10}^{-1 / 2}\left(\frac{\eta_{\mathrm{e}}}{\eta_{\mathrm{eS}}}\right)^{-1 / 2}(\mathrm{esu})$

where $L_{10}$ is the loop length scaled to $10^{10} \mathrm{~cm}$ and $\eta_{\mathrm{eS}}$ is the Spitzer electric resistivity computed for a temperature of $10^{6}$ Kelvin (Benz 1993). On the other hand, the current density produced by random footpoint motions may be limited by a maximum twisting of the magnetic field to a value of less than about

$j=\frac{c}{2 \pi} \frac{B}{L} \sim 10^{2} B_{2} L_{10}^{-1}(\mathrm{esu})$

(Aschwanden 2005), where $B_{2}$ is the longitudinal magnetic field along the loop in units of $10^{2} \mathrm{G}$. In the context of our model, we can reconcile Eqs. (8) and (9) by assuming a typical value of the resistivity in the corona

$\eta_{\mathrm{e}} \sim 10^{9} \eta_{\mathrm{eS}} \sim 10^{-8} \mathrm{~s}$

for typical values $B_{2} \sim 1$ and $L_{10} \sim 0.1$. The same rationale was used in Van Ballegooijen (1986) to estimate the small length scales of the coronal magnetic field, which produce electric currents suitable for providing the observed amount of heating.

We have no physical justification for such a high value of the coronal resistivity. Plasma phenomena, such as particlewave interactions, inertial effects or non-gyrotropic particle orbits can enhance the resistivity by up to only $10^{6}-10^{7}$ times the Spitzer resistivity locally in the solar corona. The importance of these effects is studied either with analytic estimations (Martens \& Young 1990), numerical computations (Büchner \& Elkina 2006), and laboratory experiments (Takeda \& Inuzuka 2000). Interestingly, the 3D MHD simulations of Gudiksen \& Nordlund (2002), which reproduce a typical active region, were produced with an effective (numerical) resistivity that is higher than ours. Therefore, our estimate of $\eta_{\mathrm{e}}$ remains phenomenological.

Our present paper represents a first step towards a detailed coronal-loop heating model. Given our lack of understanding of the physical mechanism producing such an enormous discrepancy between $\eta_{\mathrm{es}}$ and $\eta_{\mathrm{e}}$, we ignore any dependence of the electric resistivity on other plasma variables, such as temperature (see however Rosner et al. 1978b and Buchlin et al. 2007, who consider mechanisms such as special turbulent regimes that can occur in the solar corona).

We show that, irrespective of the presence of strong highly intermittent electric currents along the loops, the current free approximation remains valid on average. For the present calculation, $90 \%$ of the $|\delta V|$ values are in the range $10^{6}-10^{8} \mathrm{~V}$, and $90 \%$ of the mean current density along the loops are in the range $6 \times 10^{-6}-10^{-3}\left(\mathrm{~A} / \mathrm{m}^{2}\right)$. Along each loop, we computed the $\alpha=\nabla \times B / B$ parameter. For the present computation, $90 \%$ of the $\alpha$ values are in the range of $\pm\left(10^{-3}-0.1\right)\left(\mathrm{Mm}^{-1}\right)$. Since the electric currents are orientated randomly along each 


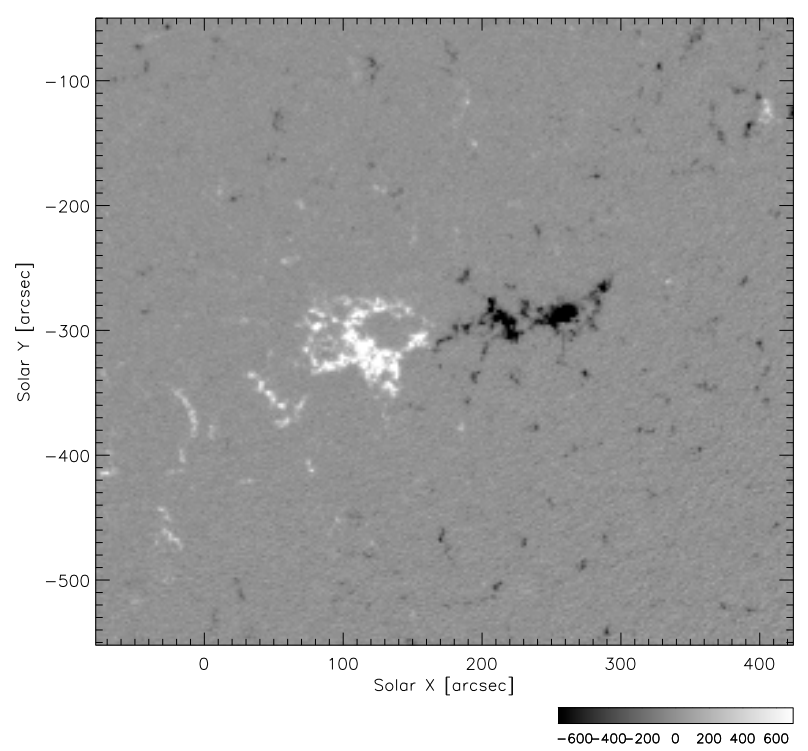

Fig. 1. Part of the MDI magnetogram of the active region NOAA 9366 that was used for the magnetic field extrapolation. White (Black) stands for positive (negative) line of sight photospheric magnetic fields. The active region is characterized by two dominant polarities.

loop, $\alpha$ switches from positive to negative values in neighboring positions. Therefore, the mean $\alpha$ measured over a bunch of loops will be close to zero, which is consistent with the potential field hypothesis. For example, in the area centred on $(x, y)=\left(138^{\prime \prime},-315^{\prime \prime}\right)$ (see Fig. 1) of the magnetogram, the average value of $\alpha$, as calculated over 360 current carrying loops is $0.003 \mathrm{Mm}^{-1}$ with a standard deviation of $0.026 \mathrm{Mm}^{-1}$. We conclude that the potential approximation could be valid on large scales, but not on small scales (lower than a few arcseconds), where the magnetic field may be highly non-potential.

\section{Scaling laws}

The present model was applied to the active region NOAA 9366, which was observed by the Michelson Doppler Imager (MDI) magnetograph (Scherrer et al. 1995) and the Extreme Ultraviolet Imaging Telescope (EIT), (Delaboudinière et al. 1995) both onboard the SOlar and Heliospheric Observatory (SOHO) satellite (Domingo et al. 1995), at (S25W11) on the Sun, on March 6, 2001. This active region was selected because of its low level of activity (absence of flares), and isolation from neighboring active regions. The magnetogram shows two spots of opposite polarity, and no detected parasitic polarities (Fig. 1). The spatial resolution of the magnetogram is $2^{\prime \prime}$, which corresponds to about $1430 \mathrm{~km}$ at the surface of the sun. As we said, a potential magnetic field extrapolation is a first order approximation of the magnetic field structure above the active region. Since we focus on a statistical description of the numerical results computed by our model, we note that this approximation is sufficient (see, however, a discussion about the coronal loop cross-section expansion with height in Sect. 5). We apply the method presented by Alissandrakis (1981), by taking into account the geometric corrections of the longitudinal magnetic field due to the position of the active region on the solar disk. The result of our extrapolation is plotted in Fig. 2.

We consider field lines originating in each one of the MDI measurement points. We select only field lines for which $\left|B_{z}\right|>$ 16 Gauss at both footpoints (Mandrini et al. 2000) with length larger than 2 pixels. This reduces the MDI noisy measurements

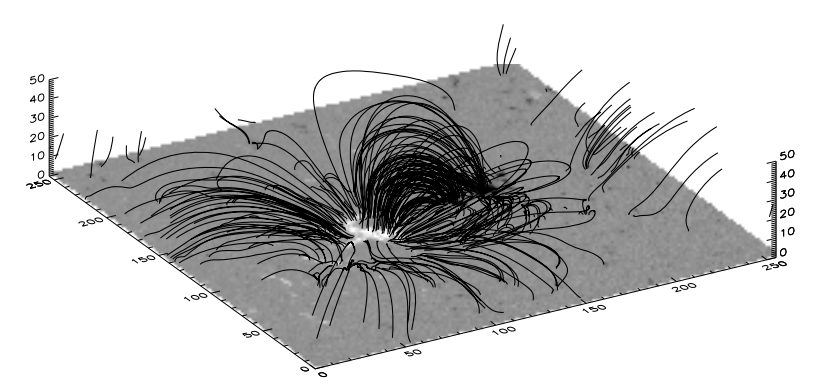

Fig. 2. Field lines of the magnetic field extrapolation that were chosen to represent the active region loops. Most of the loops connect the two dominant polarities. The axes represent the pixel size of the magnetograph.

of the magnetic field (Liu \& Norton 2001). Field lines that cross the upper boundaries of the computation box are not shown, and given the magnetogram spatial resolution, this produced 1500 field lines. In our present analysis, these field lines trace coronal loops with the cross-section equal to the MDI spatial resolution squared at one footpoint, i.e. $(1430 \mathrm{~km})^{2}$.

To study the statistical properties of the active region above the magnetogram shown in Fig. 2, we calculate the cross section (Eq. (4)), the resistance $R$ (Eq. (3)), the electric current (Eq. (5)), and the volumetric heating (Eq. (6)), for each one fo the 1500 coronal loops obtained above. Some important statistical characteristics, and parameters of the heating model are shown in Fig. 3. Panels a) and b) show the scatter plot of the mean loop magnetic field $\bar{B}$ and mean loop magnetic field energy density $\overline{B^{2}}$ as a function of the loop length. The parts of the two scatter plots between 50 and $100 \mathrm{Mm}$ can be represented with power laws of the form

$\bar{B} \propto L^{-1.4}$, and $\overline{B^{2}} \propto L^{-2.2}$.

Our least squares fit appears to be on the edges of the distributions presented in Mandrini et al. (2000). Panel c) shows the scatter plot of the loop maximal cross section as a function of the loop length. Longer loops reach higher altitudes, where the magnetic-field strength is smaller and the cross section larger. Panel d) shows the scatter plot of the resistance as a function of the loop length. The resistance has an almost linear dependence on the loop length for small loops around 3-50 Mm. However, for longer loops, the resistance decreases due to the larger cross sections. The resistance maximal value is of the order of $0.2 \mathrm{Ohm}$ for loops of roughly $100 \mathrm{Mm}$. Panel e) illustrates the dependence of the mean volumetric heating $h$ on the loop length $L$. The scatter plot indicates a power law

$\bar{h} \propto L^{-1.5}$.

This result is compatible with the index of $-2 \pm 1$ deduced from observations (Porter \& Klimchuk 1995). Finally, in panel f), we show the scatter plot of the mean volumetric heating with the mean magnetic energy density

$\bar{h} \propto \overline{B^{2}}$.

We observe that the heating is roughly proportional to the magnetic field energy.

An important characteristic of the computed volumetric heating function is its distribution with height. In Fig. 4, we see that the mean volumetric heating decreases with height in the same way as the mean magnetic energy density (dashed line). 

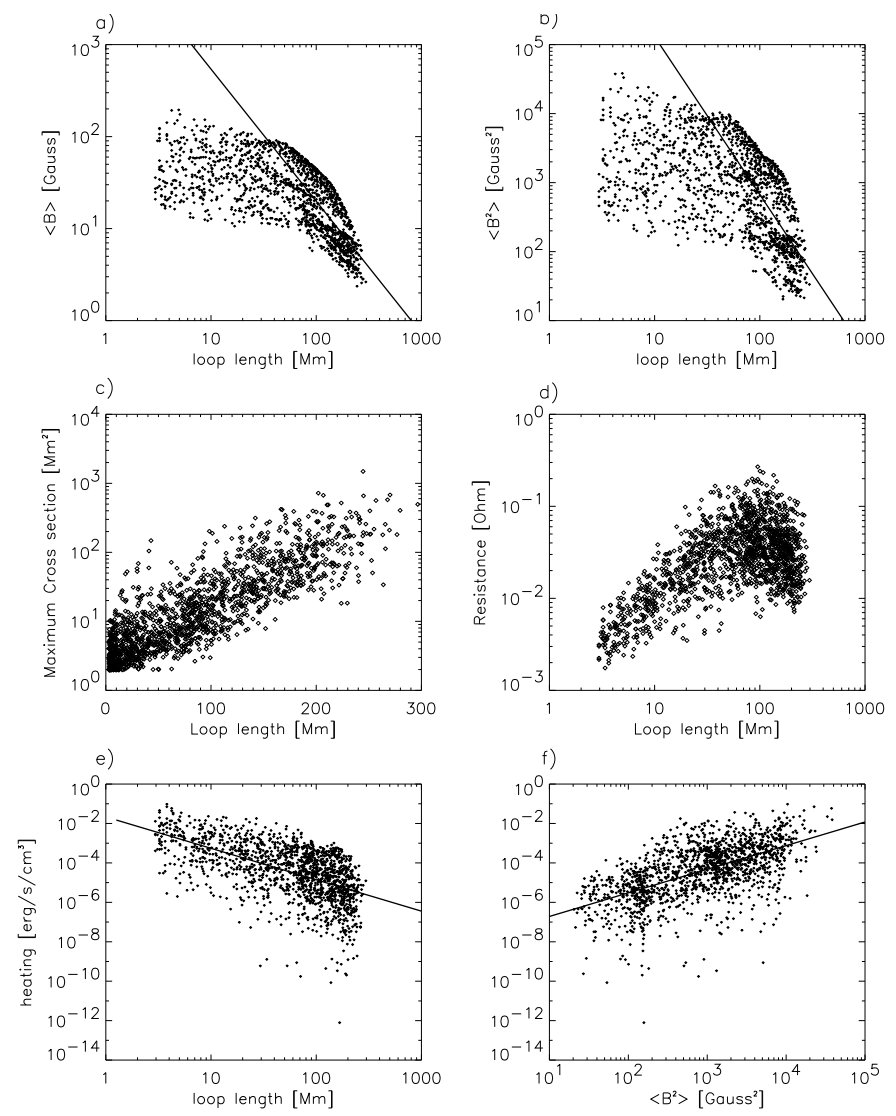

Fig. 3. Averages of the magnetic field (panel a), the magnetic field energy (panel b), the maximal loop cross section (panel c), the resistance (panel d), and the average volumetric heating (panel $\mathbf{e}$ ), as a function of the loop lengths. Panel f) shows the scatter plot between the mean volumetric heating and the mean magnetic energy along the loops. In the first two panels, a linear fit, in logarithmic unit was completed for loop lengths in the range 50-100 Mm.

This is expected because the heating is roughly proportional to the magnetic field energy density (Fig. 3f), and, both quantities drop by almost an order of magnitude in the first $10 \mathrm{Mm}$ above the photosphere. We note that our result disagrees with detailed numerical simulations (e.g. Gudiksen \& Nordlund 2002).

In Fig. 5 we show the scatter plot of the loop maximum width versus the average heating of the loops. We note that we consider only loops that extend above the chromosphere, i.e. that are higher than about $2.5 \mathrm{Mm}$. The width enhancement is defined as the square root of the ratio of the maximum cross section at the loop top over the average footpoint cross section at the level of the chromosphere. We separate the loops in two scatter plots according to their length: $14-70 \mathrm{Mm}$ in panel a), and 70-220 Mm in panel b). According to Eq. (7), only loops with heating rates above $10^{-3} \mathrm{erg} \mathrm{cm}^{-3} \mathrm{~s}^{-1}$ correspond to observable coronal structures. We can directly see from panel a) that these loops have a maximum expansion on the order of about 2 . As seen in panel $b$ ), loops with larger expansion factors have a lower heating rate and are therefore not observable.

The heating along the loops is related to the magnetic field observed at their footpoints. Figure 6 shows the scatter plot of the heating flux density through the footpoints $F$ (in $\mathrm{erg} \mathrm{cm}^{-2} \mathrm{~s}^{-1}$ ) as a function of the average of $B_{0 \perp}$ at both footpoints. This scatter plot can be fitted by the relation

$F \propto B_{0 \perp}^{1.74}$

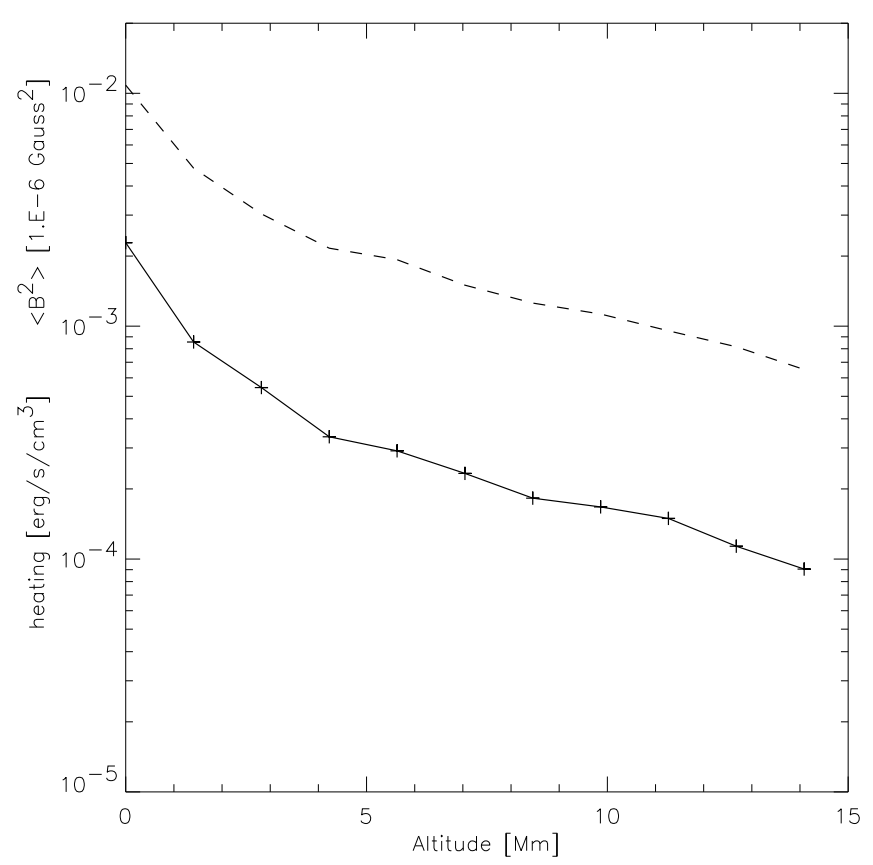

Fig. 4. Mean volumetric heating (solid line) and mean magnetic field square (dashed line) as functions of height above the active region shown in Fig. 1.
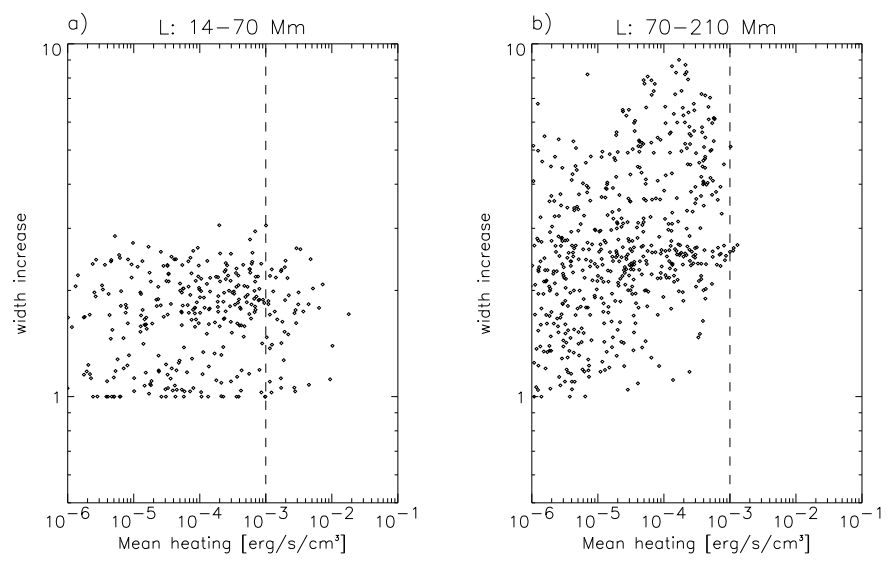

Fig. 5. Maximum loop width (cross section square root) versus mean heating along the loops. The loop points are separated into 2 panels according to their length.

which is compatible with an index of $1 \pm 0.5$ measured from observations (Schrijver \& Aschwanden 2002).

A final note about the loop geometry is in order here. Coronal loops are highly asymmetric and, as we see, this affects the heating function in a significant way. To quantify this asymmetry, we divided the loops into two parts of unequal length around their apex. For the two parts, we compared the heating length scale (defined as $h /|\mathrm{d} h / \mathrm{d} s|)$ and the heating $h$ at the footpoints. As before, we considered only the coronal part of the loop above $2.5 \mathrm{Mm}$. Our results are shown in Fig. 7. The geometry of 4 characteristic loops is shown in panel c), and their corresponding heating functions $h(s)$ in panel d). In panel a), we plotted the ratios of the two heating length scales over the loop length. Panel b) shows the footpoint heatings for each loop. We see that heating length scales and footpoint heating, can vary over several orders of magnitude from one footpoint to the other, for the 


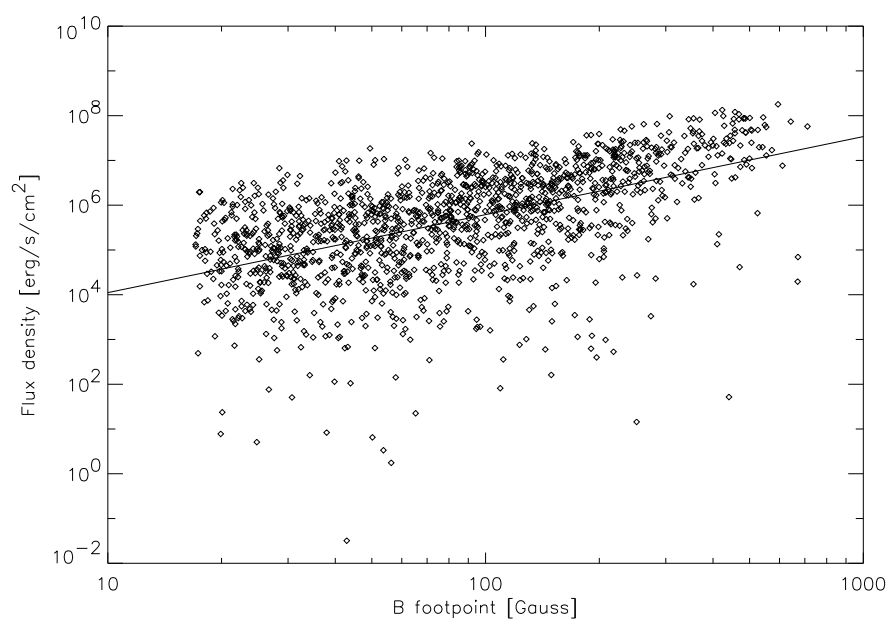

Fig. 6. Heating flux as a function of the average loop footpoint normal magnetic field. Each diamond in this plot represents a single loop.

same loop. Fewer than 30 loops are quasi-symmetric and are represented close to the diagonals of panels a) and b).

\section{Hydrostatic equilibrium}

It is known that loops with asymmetric heating functions develop unidirectional mass flows from the most heated footpoint to the less heated one (Winebarger et al. 2002; Patsourakos et al. 2004). One can nevertheless obtain a first approximation to the scaling relations of the full hydrodynamic problem by calculating hydrostatic loop atmospheres along only one half of the loop (Mok et al. 2005). We therefore decided to solve the energy and pressure balance equations

$$
\begin{aligned}
& \frac{\mathrm{d}}{\mathrm{d} s}\left\{S \kappa_{0} T^{5 / 2} \frac{\mathrm{d} T}{\mathrm{~d} s}\right\}=S\left\{h-n_{\mathrm{e}}^{2} \operatorname{Rad}(T)\right\} \\
& \frac{\mathrm{d} p}{\mathrm{~d} s}=\mu m_{\mathrm{H}} n_{\mathrm{e}} g_{\odot} \cos \theta
\end{aligned}
$$

from the footpoint to the top of each loop, where $S, T, h$, $n_{\mathrm{e}}$, and $p$ are the loop cross section, temperature, volumetric heating, electron density, and pressure along the loop, respectively. The pressure, temperature, and electron density are related by the ideal gas law as $p=2 n_{\mathrm{e}} k_{\mathrm{B}} T$, where the factor two is included because of the fully ionized plasma conditions and $k_{\mathrm{B}}$ is the Boltzmann constant. In the energy balance equation, Eq. (15), the left hand side term is the thermal conduction for ionized gases (Spitzer 1962) along the loop, where $\kappa_{0}=9.2 \times 10^{-7} \mathrm{erg} \mathrm{s}^{-1} \mathrm{~cm}^{-1} \mathrm{~K}^{-7 / 2}$.

The use of classical thermal conductivity is justified by the analysis of damped acoustic waves observed along coronal loops (Ofman \& Wang 2002; Klimchuk et al. 2004). The right hand side takes into account volumetric heating and radiative cooling. The latter is proportional to the product of the square of the electric density and a function of the temperature $\operatorname{Rad}(T)$, which is given by piecewise power laws (Rosner et al. 1978a). In the pressure balance equation, Eq. (16), $\mu=1.257$ is the average ion mass for a coronal composition of a $90 \%$ hydrogen and $10 \%$ helium plasma (Aschwanden \& Schrijver 2002), $m_{\mathrm{h}}=1.67 \times 10^{-24} \mathrm{~g}$ is the hydrogen mass, $g_{\odot}=2.74 \mathrm{~cm} \mathrm{~s}^{-2}$ is the gravitational acceleration on the solar surface, and $\theta$ is the angle between the gravitational force and its projection along the given coronal loop.
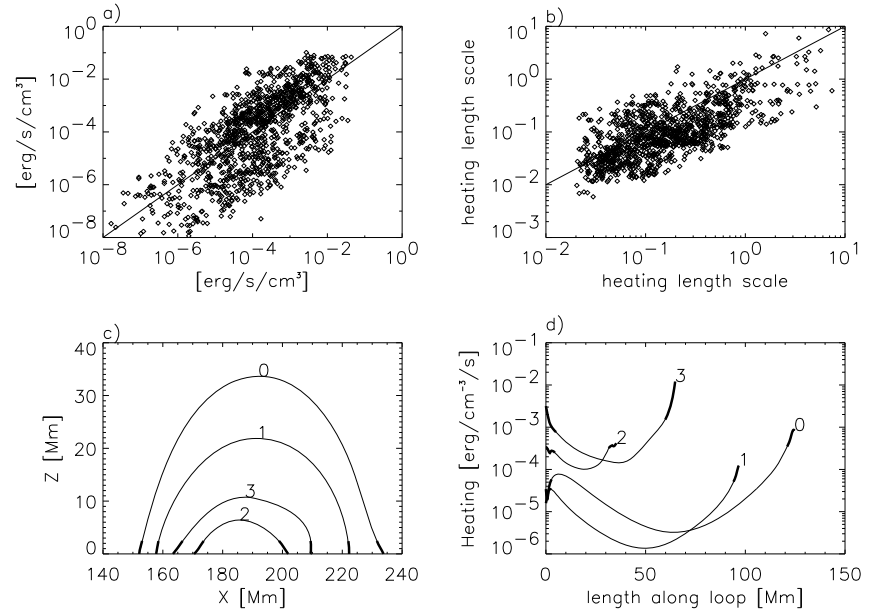

Fig. 7. Statistics of the loop heating asymmetry are shown in the panels of the first row. Panel a) shows the ratios of the heating length scales over loop lengths. Panel b) shows the heating rate at each footpoint. Four examples of loops geometry (panel c) and heating (panel d) along their length showing their asymmetry. The thick parts of the curves correspond to the chromosphere.
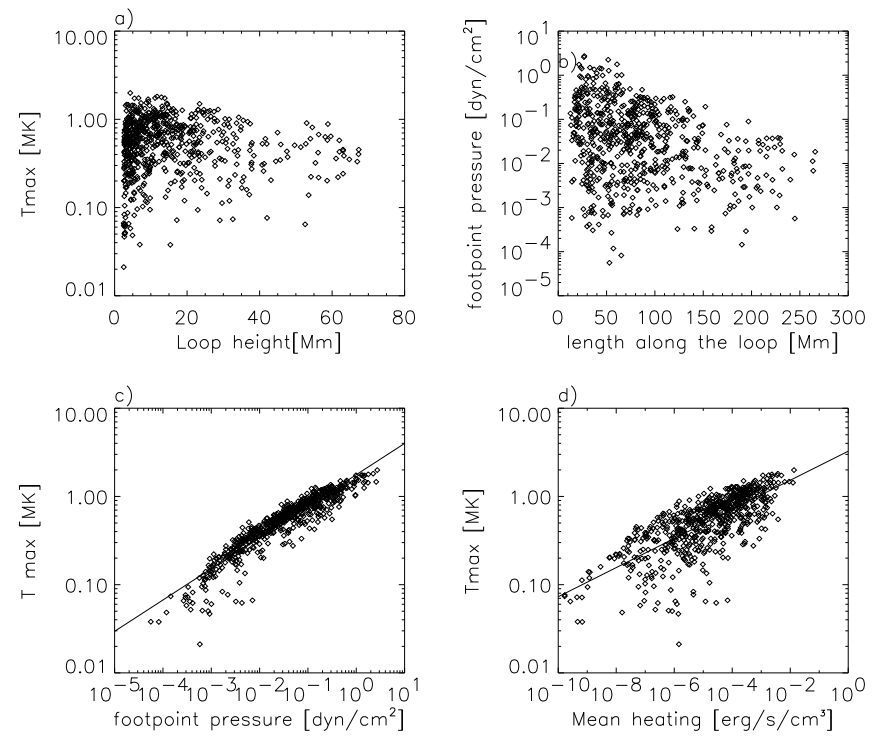

Fig. 8. Correlations derived from the stable hydrostatic solutions for half loops. Panel a) shows the maximum temperatures as a function of the loop lengths. Panel b) shows the footpoint pressure as a function of the loop lengths. Panel c) shows the maximum temperature as a function of the footpoint pressure and panel d) the maximum temperature as a function of the mean heating.

The integration starts at an altitude of $2.5 \mathrm{Mm}$, above the chromosphere and ends at the top of the loop. The boundary conditions at the coronal footpoint consist of temperature of $2 \times$ $10^{4} \mathrm{~K}$ (Aschwanden \& Schrijver 2002; Rosner et al. 1978a) and zero conduction, which implies that the loops are in equilibrium and do not conduct energy to and from the chromosphere. For each loop, the base pressure of the solution is obtained by means of a "shooting" method that guarantees $\mathrm{d} T / \mathrm{d} s=0$ at the top of the half loop.

Figure 8 shows scatter plots derived from the stable hydrostatic half-loop solutions. Panel a) shows the maximum temperatures as a function of the loop heights: $90 \%$ of the loop 
heights are below $35 \mathrm{Mm}$, and $90 \%$ of the hottest loops, above $800000 \mathrm{~K}$, have a height below $26 \mathrm{Mm}$. In panel b), the loop base pressures are in the $10^{-4}-1 \mathrm{dyn} / \mathrm{cm}^{2}$ range. The higher temperatures and base pressures are found for shorter loops. The maximum loop temperatures and base pressures, as seen in panel c), are related by a power law

$T_{\max } \propto p_{0}^{0.35}$.

Finally, as seen in panel d), the maximum temperatures are well correlated with the total volumetric heating of the loops as

$T_{\max } \propto h^{0.16}$.

The particular heating function and loop geometry of our model correspond to specific values of the above power-law exponents, which differ from those derived for hydrostatic solutions (e.g. Rosner et al. 1978a). The tendency for low lying loops to be hotter is, however, in agreement with observations (Mason et al. 1999).

We simulated and compared the active region morphology with two images of the active region recorded with the EIT telescope taken in the $171 \AA$ and $284 \AA$ passbands. We then computed the brightness of the hydrostatic loops in the $171 \AA$ and $284 \AA$ passbands using the response functions of EIT. We took into account the loop width when computing the emission images. In Fig. 9 we compare the two EIT images of the active region with the corresponding simulated images. The two EIT images were taken within $2 \mathrm{~h}$ of the magnetogram observation time. The intensities in the four panels are in a logarithmic scale.

By comparing panels a) and c) of Fig. 9 we can see that the magnetic field extrapolation represents qualitatively a class of loops connecting the two main magnetic polarities of the active region with their east footpoint at $\left(90^{\prime \prime},-310^{\prime \prime}\right)$ and their west one at $\left(250^{\prime \prime},-305^{\prime \prime}\right)$ in solar coordinates. In the 171 EIT image, there are also loops with one footpoint anchored at $\left(90^{\prime \prime},-310^{\prime \prime}\right)$ a second footpoint in the east part of the active region and loops anchored at $\left(250^{\prime \prime},-305^{\prime \prime}\right)$ closing down at the west part of the active region. In panel c), these "periphery" loops are represented by field lines that cross the boundaries of the computational box. The west footpoints of these " east periphery" field lines have scattered positions far away from the pixels corresponding to $\left(90^{\prime \prime},-310^{\prime \prime}\right)$ in the EIT image. A possible reason is that we omitted field lines crossing the high altitude boundary of our computational box (open lines). In conclusion, we can say that the magnetic field extrapolation represents qualitatively some of the main morphological structures of the active region, although it is not sufficiently accurate for a detailed comparison with observations. Even with this limitation, we obtained some interesting results on the computed active region morphology from the two passband images.

By comparing the EIT images a) and b) we can see that the bunches of loops in the 171 passband which represent cooler $\left(10^{6} \mathrm{~K}\right)$ plasmas, cover a larger area than the hottest $(1.5-2 . \times$ $10^{6} \mathrm{~K}$ ) loops in the $284 \AA$ image. This is more obvious for the "periphery" loop system, which is hardly visible in the case of the 284 EIT image. Panels c) and d) show a similar morphology. The "periphery" computed loops that cross the borders in panel c) are less visible in panel d), which means that on average they are far cooler. The reason is that only 41 of the computed loop atmospheres have a maximum temperature higher than $1.25 \times 10^{6} \mathrm{~K}$. These loops connect the main polarities in the central core of the active region and appear mostly in the $284 \AA$ passband EIT image. In these panels we can also see that the bright loops appearing in the computed image have a
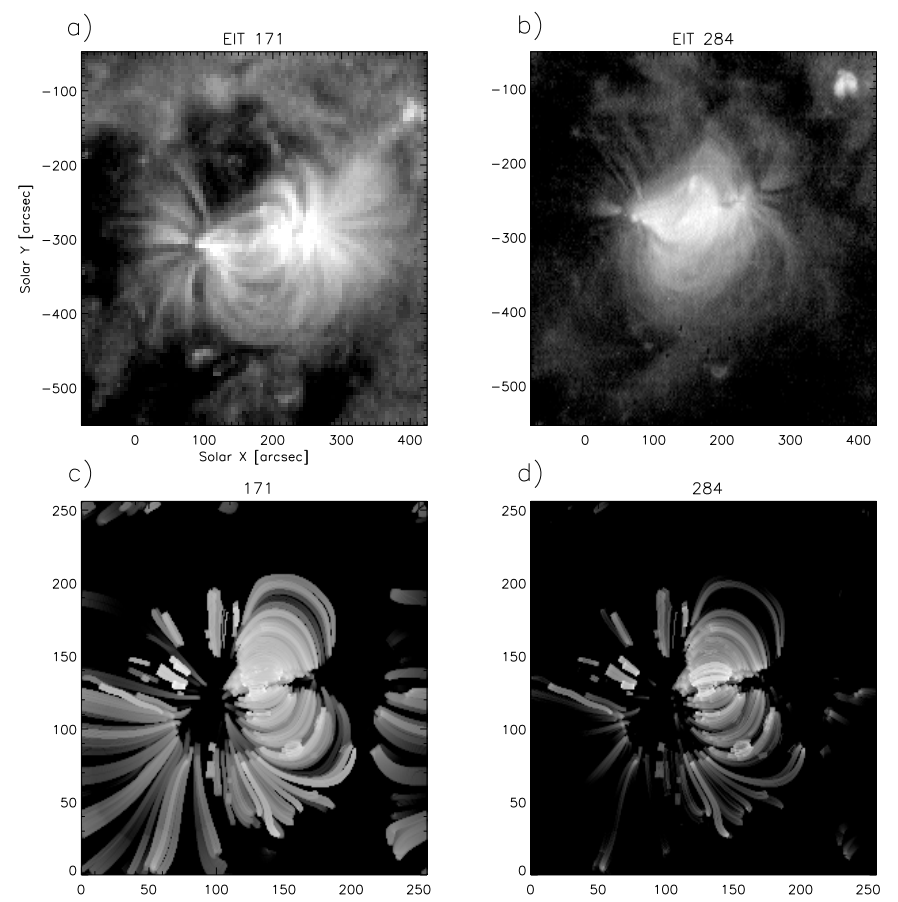

Fig. 9. Panels a) and b) show AR 9366, observed in the $171 \AA$ and $284 \AA$ EIT passbands respectively. Panels c) and d) show the computed emission in 171 and 284 based on the hydrostatic solution loops.

small thickness variation along their length. In fact, the heatingmaximum thickness relation of Fig. 5 is stronger for the loops with a hydrostatic solution so that the brighter loops have a smaller thickness variation.

\section{Discussion and conclusions}

We have presented a simple model for the distribution of coronal heating produced by DC-currents along coronal magnetic-field lines. The electric currents are due to the potential differences at the footpoints of the magnetic field lines induced by photospheric plasma motions. In our picture, heated plasma along magnetic field lines appears as bright coronal loops. We applied our picture to a particular observed active region. Coronal loops were simulated using a potential extrapolation of the magnetic field observed at the level of the photosphere with MDI. We calculated the electric resistance of each loop and applied Ohm's law to obtain the heating function at each point of the active region. Given the heating function and magnetic field geometry, only a small fraction of the coronal loops support a hydrostatic atmosphere.

Our model yields several testable results, expressible as scaling laws that could be compared with observations. An important result is the heating-loop length relation represented by a power law in Eq. (12). This result is compatible with the index of $-2 \pm 1$ deduced from observations (Porter \& Klimchuk 1995). Moreover, the heating that we obtain is closely proportional to the square of the magnetic field along the loop (Fig. 3f), which implies that the mean heating of the active region variation with altitude is similar to the variation in the magnetic energy. Our result differs from that of Gudiksen \& Nordlund (2002), where the heating decreases more sharply with altitude than $B^{2}$. Finally, the heating-flux density versus footpoint magnetic-field relation described by the relation in Eq. (14), is compatible with the results of Schrijver \& Aschwanden (2002), which were deduced 
from observations. The expressions of the loop heating distributions presented in Eqs. (12) and (14) correspond to the particular morphology of the active region, where the hottest loops are the shortest ones that connect the two main magnetic-field polarities of the active region (Fig. 9). These loops dominate in the simulated $284 \AA$ passband of the EIT instrument whereas cooler loops, which do not connect only the two main magnetic-field polarities, are mostly detectable in the 171 passband in good agreement with observations. Despite its simplicity, we therefore conclude that our model is able to reproduce the observations.

An interesting yet unsolved problem is that coronal loops appear to have a constant width along their length. The largest measured width variation is 1.15 to 1.3 from footpoint to loop top (Klimchuk et al. 1992; Aschwanden et al. 1999; Watko Klimchuk 2000; Lopez Fuentes et al. 2006). In the present simulation, only loops with a small width expansion are heated to an observable heating rate (Fig. 5). The expansion factors obtained for those loops are slightly higher than the aforementioned observed values. A possible resolution of this inconsistency could be to include the random longitudinal (i.e. along the loop) electric currents of our model in a more accurate non-potential reconstruction of the active region magnetic field (see however Lopez Fuentes et al. 2006). We expect that the higher the longitudinal electric current density, the more uniform the loop cross section along its length.

The loops and the associated heating functions have a high degree of asymmetry. Such loops cannot support atmospheres in hydrostatic solutions and will develop mass flows. To a first order approximation, we can derive the temperature and density distribution along the loop by computing hydrostatic solutions for one half of the loop, from one footpoint to the loop's apex.

The hotter of these loops are found at lower altitudes (Fig. 8), which is compatible with observations. Other physical processes such as steady MHD flows (Petrie 2006b), or impulsive heating (see Patsourakos \& Klimchuk 2006; Warren \& Winebarger 2006), may also be considered in the calculation of the structure of the active region atmosphere.

We conclude by stating once again that our model presents a simple idea that may answer some aspects of the coronal heating without complex numerical simulations. Several possibilities, such as the non-potential nature of the magnetic field extrapolation, variable electric resistivity, more realistic photospheric velocity field, and non hydrostatic loops, may further be explored in future work.

Acknowledgements. We would like to thank our anonymous referee who helped to improve our manuscript, as well as S. Patsourakos and G. J. D. Petrie for useful suggestions. CG dedicates this work to the memory of Stephanos C. Gontikakis.

\section{References}

Alissandrakis, C. E. 1981, A\&A, 100, 197

Aschwanden M. J. 2005, in Physics of the Solar Corona (Berlin: Springer)

Aschwanden M. J., \& Schrijver C. J. 2002, ApJS, 142, 269

Aschwanden M. J., Newmark, J. S., Delaboudiniére, J.-P., et al. 1999, ApJ, 515, 842

Benz A. 1993, in Plasma Astrophysics, Kinetic processes in Solar and Stellar Coronae (The Netherlands: Kluwer Academic Publishers)

Buchlin, E., Cargill, P. J., Bradshaw S. J., \& Velli, M. 2007, A\&A, 469, 347

Delaboudinière, J. P., Artzner, G., Brunaud, J., et al. 1995, Sol. Phys., 162, 291 Büchner, J., \& Elkina, N. 2006, Phys. Plasmas, 13, 082304

Domingo, V., Fleck, B., \& Poland, A. I. 1995, So., 162, 1

Dmitruk P., \& Gómez D. O. 1997, ApJ, 484, 83

Dmitruk P., \& Gómez D. O. 1999, ApJ, 527, 63

Fisher, G. H., Longcope, D. W., \& Metcalf, T.R. 1998, ApJ, 508, 885

Golub L., Max n C., Rosner R., Serio S., \& Vaiana G. S. 1980, ApJ, 238, 343

Gudiksen, B. V., \& Nordlund 2002, A\&A, ApJ, 572, L113

Gudiksen, B. V., \& Nordlund 2005a, A\&A, ApJ, 618, 1020

Gudiksen, B. V., \& Nordlund 2005b, A\&A, ApJ, 618, 1031

Hendrix, D. L., Van Hoven, G., Mikic, Z., \& Schnack, D. D. 2001, ApJ, 470, 1192

Klimchuk, J. A., \& Porter L. J. 1995, Nature, 377, 131

Klimchuk, J. A. 2006, So., 234, 41

Klimchuk, J. A., Lemen, J. R., Feldman, U., Tsuneta, S., \& Uchida, Y. 1992, PASJ, 44, 181

Klimchuk, J. A., Tanner, S. E. M., \& De Moortel, I. 2004, ApJ, 616, 1232

Lopez-Fuentes, M. C., Klimchuk, J. A., \& Démoulin, P. 2006, ApJ, 639, 459

Liu, Y., \& Norton, A.A. 2001, SOI-Technical Note 01-144

Mandrini C. H., Demoulin, P., \& Klimchuk, J. A. 2000, ApJ, 530, 999

Martens, P. C. H., \& Young, A. 1990, ApJS, 73, 333

Mason, H. E., Landi, E., Pike, C. D., \& Young, P. R. 1999, Sol. Phys., 189, 129

Mok, Y., Mikic, Z., Lionello, R., \& Linker, J. A. 2005, ApJ, 621, 1098

Ofman, L., \& Wang, T. 2002, ApJ, 580, 85

Parker, E. N. 1972, ApJ, 174, 499

Patsourakos, S., \& Klimchuk, J. A. 2006, ApJ, 647, 1452

Patsourakos, S., Klimchuk, J. A., \& MacNeice, P. J. 2004, ApJ, 603, 322

Petrie, G. J. D. 2006a, ApJS, 166, 378

Petrie, G. J. D. 2006b, ApJ, 649, 1078

Porter, L. J., \& Klimchuk, J. A. 1995, ApJ, 454, 499

Rappazo, A. F., Velli, M., Einaudi G., \& Dahlburg R. B. 2007, ApJ, 657, 47

Rosner, R., Tucker W. H., \& Vaiana G. S. 1978a, ApJ, 220, 643

Rosner, R., Golub, L., Coppi, B., \& Vaiana G. S. 1978b, ApJ, 222, 317

Scherrer, P. H., Bogart, R. S., Bush, R. I., et al. 1995, Sol. Phys., 162, 129

Schrijver, C. J., \& Aschwanden, M. J. 2002, ApJ, 566, 1147

Schrijver, C. J., Sandman, A. W., Aschwanden, M. J., \& DeRosa, M. L. 2004 ApJ, 615, 512

Serio, S., Peres G., Vaiana G. S., Golub L., \& Rosner R., 1981, ApJ, 243, 288

Spitzer Jr. L. 1962, Physics of Fully Ionized Gases (New York: Inter Science)

Takeda, Y., \& Inuzuka H. 2000, Phys. Lett. A, 265, 282

Van Ballegooijen, A. A. 1986, ApJ, 311, 1001

Warren H. P., \& Winebarger A. R. 2006, ApJ, 645, 711

Watko J. A., \& Klimchuk, J. A. 2000, So., 193, 77

Winebarger A. R., Warren H. P., Van Ballegooijen A., DeLuca, E. E., \& Golub, L. 2002, ApJ, 567, 89

Withbroe, G. L, \& Noyes, R.W. 1977, ARA\&A, 15, 363 